Research Article

\title{
The Impact of Migration on Psychosocial Well-Being: A Study of Student Migrants in Resettlement State
}

\author{
Vanshika Thukral', Veena Sharma ${ }^{2}$, Jamal Fatima ${ }^{3}$
}

${ }^{1}$ M.Sc. Nursing Student, ${ }^{2}$ Associate Professor, ${ }^{3}$ Assistant Professor, Rufaida College of Nursing, Jamia Hamdard, New Delhi. DOI: https://doi.org/10.24321/2455.9318.202009

\section{I $\quad \mathbf{N} \quad \mathbf{F} \quad \mathbf{O}$}

\author{
Corresponding Author: \\ Vanshika Thukral, Rufaida College of Nursing, \\ Jamia Hamdard, New Delhi \\ E-mail Id: \\ vanshikathukral27@gmail.com \\ Orcid Id: \\ https://orcid.org/0000-0001-8374-5996 \\ How to cite this article: \\ Thukral V, Sharma V, Fatima J. The Impact of \\ Migration on Psychosocial Well-Being: A Study \\ of Student Migrants in Resettlement State. Int J \\ Nurs Midwif Res 2020; 7(1): 48-52.
}

Date of Submission: 2020-04-15

Date of Acceptance: 2020-05-27

\section{$\begin{array}{llllllll}\mathbf{A} & \mathbf{B} & \mathbf{S} & \mathbf{T} & \mathbf{R} & \mathbf{A} & \mathbf{C} & \mathbf{T}\end{array}$}

Introduction: The stresses of the migration process when combined with a lack of social support, a discrepancy between achievement and expectations, economic hardships, racial discrimination and harassment, and a lack of access to proper housing, medical care, and religious practice can lead to poor psychosocial health in student migrants. Thus, the study was conducted to assess the psychosocial problems among student migrants.

Materials and Methods: A mixed method research approach with sequential explanatory research design was used. For the quantitative data, 50 student migrants studying in Rufaida College of Nursing were selected using systematic random sampling technique using selfconstructed psychosocial problem assessment scale. The data was analysed using both inferential and descriptive statistics. Fisher exact test was used to determine relationship of psychosocial problem scores with selected demographic variables. The mean, modified mean and rank order of psychosocial problems faced by student migrants were computed. For the qualitative data, focus group discussion was conducted.

Result: The study revealed that $74 \%$ of the student migrants had mild psychosocial problems. A significant association was found between the current living status of the student migrant and the psychosocial problems faced by them with $p$ value 0.011 . The content analysis of the narratives of student migrants gave the following themes: 1 ) Perceptions of student migrants related to the city of migration: 'Flavour of the city', 2) Challenges faced by student migrants during the phase of migration and how they have adapted to it: Resilience vis-a-vis Challenges and 3) Viewpoints of student migrants about the effect of migration on their mental health: Migration-Loneliness.

Conclusion: There is an immense need of sensitization of the university authorities to address the concerns of students from other states and take initiatives to overcome the gap between locals and migrants.

Keywords: Migration, Student Migrants, Psychosocial Problems 


\section{Introduction}

The process of migration conglomerates a variety of concerns on various levels. Identity issues and failure to realise expectations at personal level where as social situations like cultural shock, bereavement, and lack of cultural assimilation can produce a detrimental effect on the mental as well as psychosocial health of student migrants. ${ }^{1}$ While most individuals may make a satisfactory transition, some, as they venture out, land up into the new and unknown psychosocial territories. ${ }^{2}$

Migration is a complex process, involves a myriad of causes, experiences, cultural adjustment and stages, that influence the psychosocial health of student migrants. ${ }^{3}$ The stresses and strains of the migration process when combined with a lack of social aid, incongruity between achievement and expectations of an individual, financial hardships, racial discrimination and harassment and a lack of access to proper housing, medical care and religious practice can lead to poor black self, maladjustment, and deteriorates physical, mental and psychosocial health. ${ }^{4,5}$

\section{Objectives of the Study}

- To assess the levels of psychosocial problems faced by student migrants studying in a selected college in New Delhi.

- $\quad$ To explore discrimination experiences and prejudices faced by student migrants.

- To see the association between levels of psychosocial problems faced by student migrants and selected background variables.

\section{Research Questions}

To explore the discrimination experiences and prejudices faced by student migrants, the research questions addressed through narratives of the student migrants were:

- What were the experiences of the student migrants when they first landed in the city?

- What were the challenges that came as a part of migration and how was the process of resilience?

- What were the viewpoints of student migrants about the effect of migration on their mental health?

\section{Materials and Methods}

Research approach used in this study was mixed methods approach. Quantitative approach was adopted to assess the psychosocial problems faced by student migrants. Qualitative approach was used for the in-depth exploration of discrimination and prejudice experiences and their psychosocial stresses associated with discrimination and prejudice. Research design used in the study was sequential explanatory research design. The study lasted for nearly 3 months starting from research proposal making to collection of data to presentation of data.

To assess the psychosocial problems faced by student migrants, the psychosocial assessment scale was administered to 50 student migrants studying in Rufaida college of Nursing, Jamia Hamdard who were Indian in origin and who migrated to Delhi from their state of domicile to attain higher education and are currently living in university hostel, or as paying guest. The students staying with family and staying in Delhi NCR region were excluded.

Ethical permission was taken from Institutional Ethical Committee (IEC) of Jamia Hamdard, Delhi to conduct the research study. Written informed consent was taken from each study subject. They were assured of anonymity and confidentiality of information provided during the study. The consent also gave right to subjects to withdraw from the study at any time. Participants were verbally explained about the recording of the interview. Also, coding of the participants name in both the methods was done which ensured anonymity. Pseudonyms were used while citing subjects' statements in the narratives of the subjects.

For content validation, the tool was given to nine experts from the field of Psychiatric Nursing, Psychiatry and Clinical Psychology. The suggestions and modifications as suggested by the nine experts were incorporated and the tool was modified accordingly. The reliability of the tool was calculated using Cronbach's alpha formula and the reliability was found to be 0.80 , implying the tool was reliable.

The student migrants who met the inclusion criteria were selected using systematic random sampling technique. A

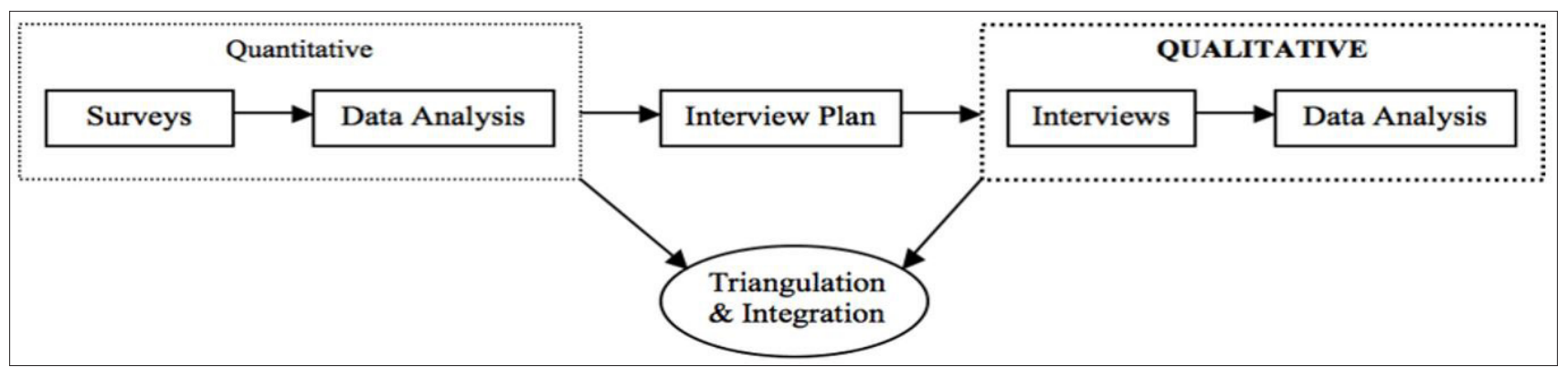

Figure I.Schematic diagram of explanatory research design 
total of 50 (n) student migrants were selected from lists of student migrants prepared from each class. ( $N=203$ ) Thus, $K$ was calculated as 4 from the formula $K=N / n$. $2^{\text {nd }}$ student in list was chosen as the first subject with the help of random table. After the first subject, every $4^{\text {th }}$ sample was selected from the list. The structured scale was divided in two parts to assess the psychosocial problems in student migrants, i.e. Part I section A: consisted of questions related to personal and family details and part I section B: consisted of items to assess the psychosocial problems in student migrants. There were 30 items to assess the psychosocial problems in the form of a 4 point rating scale with 'always', 'mostly', 'sometimes' and 'never' as a response. 'Always' was scored as 4, 'mostly' as 3, 'sometimes' as 2 and 'never' as 1.

Table I.Interpretation of scores from psychosocial problem assessment scale

\begin{tabular}{|c|c|}
\hline Scores & Levels of psychosocial problems \\
\hline $0-40$ & Severe psychosocial problems \\
\hline $41-80$ & Moderate psychosocial problems \\
\hline $81-120$ & Mild psychosocial problems \\
\hline
\end{tabular}

The table 1, is the interpretation of scores from psychosocial problem assessment scale. The scores ranging from 0-40 indicates severe psychosocial problems, 41-80 indicates moderate psychosocial problems and 81-120 indicate mild psychosocial problems. The frequency and percentage distribution table was constructed to describe the selected demographic variables of the student migrants and by their scores on psychosocial problems faced by them. The Fisher exact test was used to determine relationship of psychosocial problem scores with selected demographic variables. Further, mean, median and standard deviation of the psychosocial problems scores of student migrants was calculated and modified mean and rank order of psychosocial problems in order to rank the psychosocial problems.

Out of 50, 6 student migrants reported that they had stories to tell about discrimination and prejudice. These 6 were included in the focus group discussion to explore in-depth discrimination and prejudice experiences and psychosocial problems associated with these experiences.

The data collection tool was prepared and was divided in 3 sections: Background profile of the subjects (such as age, religion, gender, place of domicile, marital status, educational status, employment, monthly family income, type of family and information related to their migration status), structured scale to assess the psychosocial problems faced by student migrants and narratives of discrimination and prejudice experiences faced by student migrants were assessed by unstructured Focus Group Discussion. Focus group discussion was conducted for 1 hour 46 minutes on $1^{\text {st }}$ November, 2019. It was audio recorded and later transcriptions were made. Content analysis was done of the narratives of student migrants obtained through focus group discussion to form themes and subthemes.

\section{Result}

The background data of the student migrants showed that $96 \%$ of them belonged to the age group $18-22$ years and $68 \%$ of them were females. Forty percent of student migrants belonged to Uttar Pradesh, followed by J\&K (20\%) and Haryana (12\%). Nearly $90 \%$ of student migrants knew Hindi, followed by English (70\%) and Urdu (28\%). Out of 50,36 student migrants were from B.Sc. $3^{\text {rd }}$ year followed by DGNM $1^{\text {st }}$ year (11) and other classes. Nearly $3 / 4^{\text {th }}$ of student's migrants had family income of less than Rs.50, 000 . Sixty percent of student migrants belonged to a nuclear family whereas $36 \%$ belonged to a joint family. Around 40 $\%$ of student migrants migrated in the year 2019 followed by 2018 and so on. Eighty four percent migrated alone from their place of domicile for education and $40 \%$ stated poor condition of education institution, as the reason of migration. Nearly $70 \%$ of student migrants were living alone with $40 \%$ staying in university hostel and $60 \%$ staying in paying guest accommodation.

\section{LEVEL OF PSYCHOSOCIAL PROBLEMS}

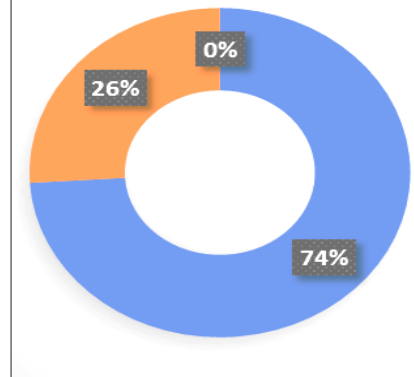

m Mild Psychosocial Problems

Moderate Psychosocial Problems

- Severe Psychosocial Problems

Figure 2.Pie diagram showing the frequency distribution of sample subjects by the severity of psychosocial problems faced by student migrants $(n=50)$

The figure 2, shows the frequency distribution of sample subjects by the severity of psychosocial problems faced by student migrants. Nearly $74 \%$ had mild psychosocial problems and $26 \%$ has moderate psychosocial problems. A significant association was found between the current living status of the student migrant and the psychosocial problems faced by them as shown in table below.

The table 2, shows the association between psychosocial problems faced by student migrants and current living status of student migrant. The value of Fischer exact test, 0.011 implies that there is significant association between psychosocial problems faced by student migrants and current living status of student migrant. 
Table 2.Association between psychosocial problems faced by student migrants and current living status of student migrant

\begin{tabular}{|c|c|c|c|c|c|}
\hline & Mild & $\begin{array}{c}\text { Mod- } \\
\text { erate }\end{array}$ & Severe & $\begin{array}{c}\text { Fischer's } \\
\text { exact } \\
\text { value }\end{array}$ & p-value \\
\hline Alone & 26 & 6 & 0 & & \\
\hline $\begin{array}{c}\text { With } \\
\text { friends/ } \\
\text { classmates }\end{array}$ & 8 & 10 & 0 & 0.011 & $0.0277^{*}$ \\
\hline
\end{tabular}

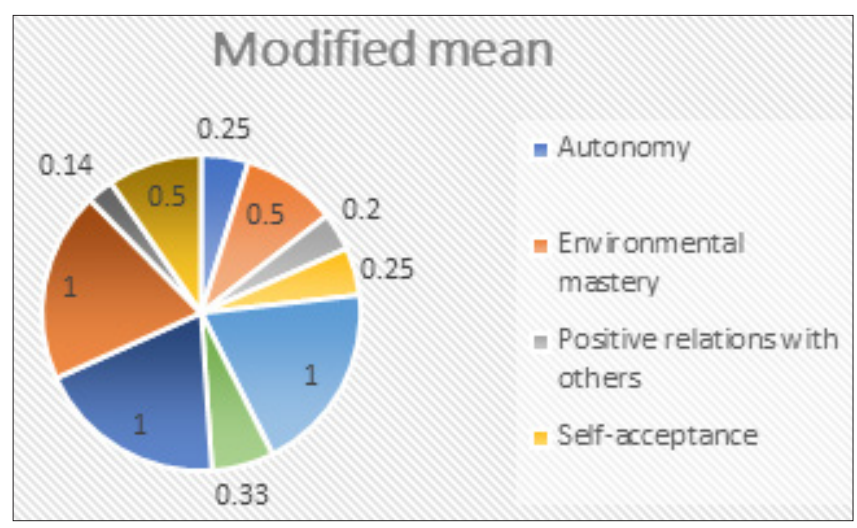

Figure 3.A pie diagram showing the area wise modified mean scores of psychosocial problems faced by student migrants

Figure 3, reveal that the maximum psychosocial problems faced were in the area of religiosity, financial constraints and linguistic barriers, followed by environmental factors and perceived social support. Grief of separation is the next in line followed by lack of autonomy and self-acceptance. As reported, the student migrants face least psychosocial problems in aspects of positive relations with others and everyday discrimination

Reflections of the student migrants: content analysis of the narratives of discrimination experiences and prejudices faced by student migrants revealed certain themes.

\section{Theme I: 'Flavour of the city?'}

The perception people portrayed are a mix of their values, earlier life experiences, social interactions and their culture as prevalent in their state of domicile. For some, it was a fearful experience while some reported a sense of feel of new beginnings in life.

A city is made up of people and they are in a reflection of the city. As Ms Fatima expressed her take on her interactions with her classmates and people in general.

"I feel that the locals are very selfish and self-centred. They only initiate talk when they need something from you. They behave strange and unknown when you need something from them. Even my classmates only talk to me when they need something from me."

\section{Theme 2: Resilience vis-a-vis Challenges}

\section{Subtheme 2.I: Cultural differences}

According to the participants' experiences, even though at the beginning both language and cultural barriers were problematic for them, language barriers eventually got reduced or faded away with time. Differences in food, clothing and language and other cultural disparities may hinder acculturation resulting in psychosocial problems.

\section{Food \& Clothing}

Five out of six student migrants reported difficulty adapting to the food and clothing style of the locals. Some reported that they stand out uncomfortably because of the way they dress while another was subjected to discriminatory comments for dressing in a particular style and choice of colour of clothes. The food habits were reported to be very different in terms of content and preparation. Even the quality of water was posed as an issue as it took them a lot of time to adapt to it.

\section{Language Barriers}

Communication in Hindi and/or English (medium of instructions being English) was a big challenge for student migrants who narrated their stories. They insisted that they would have performed much better if the lectures were in Hindi or a mix of both Hindi and English.

\section{Sub theme 2.2: Discrimination and Prejudices}

The discrimination on the basis of gender, religion and socioeconomic status was quite apparent when student migrants had to rent an accommodation. It became a challenge when the student migrants stepped out in the city and wanted to lease an accommodation or buy household stuff.

\section{Sub theme 2.3: Feelings of Insecurity}

The student migrants experienced a number of difficulties during the pre-migration and migration periods that influenced their psychological well-being in the resettlement city. Feeling of insecurity to leave their home state and be in a completely new zone. They pointed out that it was a terrible experience to leave all material and emotional belongings, without any choice; however, it was needed to survive.

\section{Sub theme 2.4: Daily Household Chores}

Two of the male participants reported doing household chores as a big challenge and time consuming task while female participants did not see it as much of a problem.

Subtheme 2.5: Blending Process-Relieving Factors

The participants said that the people from their own state 
helped them adapt to the new milieu, while three of them said that locals also helped them to adapt to the new environment, city and culture. The participants also had to alter their perception in order to tune-in with the locals.

\section{Theme 3: Migration-Loneliness}

Psychosocial distress is a by-product of migration process. It may subside with time with aid of relieving factors during blending process as discussed before. If not, can harm the psyche of the student migrant and make the one susceptible to a lot of psychological, emotional and physical issues.

\section{Discussion}

Migration is a multifaceted process. It often results in unique difficulties for the student migrants as well as their families. The complexity of the process of migration often results in psychosocial distress in student migrants. The student migrants may often feel uncertainty, anger, shame, sadness and fear.

Toselli S et al. examined the literature on the psychosocial health of student migrants in Europe and of North Africans living in their own countries and declared that migrants generally have poorer psychosocial health. The study revealed that more than half of student migrants had psychosocial issues which is line with the findings of my study which declares that $64 \%$ of the same had mild psychosocial problems. Although, in my study, a negative relation was seen between the gender and the level of psychosocial problems whereas, in his study, women generally presented a higher risk. ${ }^{11}$

Straiton ML et al. did a study to investigate the association between perceived discrimination and psychosocial health among migrants in Norway, to which he concluded that around $27 \%$ of participants reported perceived discrimination which was associated with 1.86 higher odds of psychosocial problems. Similarly, in my study, the students who narrated discrimination experiences and prejudices had low scores in psychosocial problem assessment scale indicating high level of psychosocial problems. $^{12}$

\section{Conclusion}

Majority of the student migrants had mild psychosocial problems as a result of migration. A significant association was found between the current living statuses of the student migrants with the level of psychosocial problems. Also, the student migrants reported incidents of discrimination and prejudice. There were issues related to cultural differences, safety and security, ethnic identity, loneliness. However, the students blended in and adapted to this with some selfhelp, help of locals and few people from their own states.

\section{References}

1. Virupaksha HG, Kumar A, Nirmala BP. Migration and mental health: An interface. J Nat Sci Biol Med 2014; 5(2): 233-239.

2. XPeditions NGM. NGS. Human Migration Guide (6-8). National Geographic Society, 2005; 1-5.

3. Firdaus $\mathrm{G}$. Mental well-being of migrants in urban center of India: Analysing the role of social environment. Indian J Psychiatry 2017; 59(2): 164-169.

4. Jha A, Mohammad Kawoosa V. What the 2011 census data on migration tells us. Hindustan Times, 2019.

5. World Economic Forum. Migration and Its Impact on Cities, 2019.

6. Matlin SA, Depoux A, Schütte S, et al. Migrants' and refugees' health: towards an agenda of solutions. Public Health Rev 2018; 39: 27.

7. Bhugra D, Becker MA. Migration, cultural bereavement and cultural identity. World psychiatry: Official Journal of the World Psychiatric Association (WPA) 2005; 4(1): 18-24.

8. Eisenbruch M. The cultural bereavement interview: a new clinical research approach for refugees. Psychiatr Clin North Am 1990; 13: 715-735.

9. Kuo BC. Coping, acculturation, and psychological adaptation among migrants: a theoretical and empirical review and synthesis of the literature. Health Psychol Behav Med 2014; 2(1): 16-33.

10. International Organization for Migrations. World Migration Report 2010 - The Future of Migration Building Capacities for Change. 2010. Available from: http://www.jcp.ge/iom/pdf/WMR_2010_english-pdf.

11. Toselli S, Gualdi-Russo E, Marzouk D et al. Psychosocial health among immigrants in central and southern Europe. Eur J Public Health 2014; 24(Suppl 1): 26-30.

12. Straiton ML, Aamb $\varnothing A K$, Johansen R. Perceived discrimination, health and mental health among immigrants in Norway: the role of moderating factors. BMC Public Health 2019; 19: 325.

\section{Conflicts of Interest: None}

